\title{
FLAVONOIDES Y ACTIVIDAD ANTIOXIDANTE DE Calia secundiflora (Ort.) Yakovlev
}

\author{
FLAVONOIDS AND ANTIOXIDANT ACTIVITY OF \\ Calia secundilfora (Ort.) Yakovlev
}

\section{Rosario M. Barrón-Yánez ${ }^{1}$, M. del Rosario García-Mateos ${ }^{1,2 *}$, Marcos R. Soto-Hernández ${ }^{3}$, Teresa Colinas-León ${ }^{1}$ y Geoffrey Kite ${ }^{4}$}

\begin{abstract}
${ }^{1}$ Instituto de Horticultura, Departamento de Fitotecnia, y ${ }^{2}$ Preparatoria Agrícola, Universidad Autónoma Chapingo. km 38.5 Carr. México-Texcoco. 56230, Chapingo, Edo. de México. Tel. (01) 5959521500 Ext. 5797. ${ }^{3}$ Programa de Botánica, Colegio de Postgraduados-Campus Montecillo. km 36.5. Carr. MéxicoTexcoco. 56230, Montecillo, Texcoco, Edo. de México. ${ }^{4}$ Royal Botanical Gardens Kew. Richmond, Surrey, TW9 3AB, UK.
\end{abstract}

${ }^{*}$ Autor para correspondencia (rosgar08@hotmail.com )

\section{RESUMEN}

La posible extinción de algunas especies no estudiadas como Calia secundiflora (Ort.) Yakovlev significa la pérdida del potencial que representa el conocimiento de sus metabolitos con propiedades medicinales y de interés agronómico. Los estudios fitoquímicos en C. secundiflora (Ortega) Yakovlev (Fabaceae) se han enfocado hacia los alcaloides, pero aún se desconoce su perfil de flavonoides. Estos metabolitos presentes en los vegetales se asocian con una capacidad antioxidante natural, y que estudios epidemiológicos han mostrado efectos benéficos para la salud humana. El objetivo de esta investigación fue cuantificar el contenido de compuestos fenólicos y flavonoides, así como identificar las estructuras de los flavonoides en hoja y evaluar el potencial antioxidante de los extractos. La actividad antioxidante se evaluó por el método de DPPH (2,2-difenil-1-picrilhidracilo) en muestras de una zona del Estado de Hidalgo, México. El análisis por CL-EM permitió identificar siete glicósidos en los flavonoles quercetina, isoramnetina y kaenferol, no descritos previamente para esta especie. El contenido de compuestos fenólicos totales $\left(8.31 \pm 0.38 \mathrm{mg} \mathrm{g}^{-1} \mathrm{de}\right.$ MS) fue mayor al de flavonoides (3.08 $\pm 0.32 \mathrm{mg} \mathrm{g}^{-1}$ de MS). Estos resultados explican la actividad antioxidante $\left(\mathrm{CI}_{50}=88.54 \pm 0.15 ; 109.44 \pm 0.48 \mu \mathrm{g} \mathrm{mL}^{-1}\right)$ que presentaron los extractos de hoja. La identificación de estos flavonoides puede contribuir a la quimiotaxonomía del género.

Palabras clave: Calia secundiflora, colorín, actividad antioxidante, flavonoides.

\section{SUMMARY}

The possible extinction of some non-studied species such as Calia secundiflora might mean the loss of the knowledge about their metabolites with medicinal properties and agronomic interest. The phytochemical studies on C. secundiflora (Ort.) Yakovlev (Fabaceae) have been focused on its content of alkaloids, but its profile of flavonoids is still unknown. These plant metabolites have been associated to a natural antioxidant capacity which are beneficial for human health according to epidemiological studies. The objective of this research was to quantify the contents of phenolic compounds and flavonoids, as well as to identify the flavonoid structures in leaf and to evaluate their antioxidant potential. The antioxidant activity was evaluated by the DPPH (2,2-diphenyl-1-picrylhydrazyl) method in samples taken from Hidalgo State, México. The analysis by HPLC-MS allowed to the identify seven glycosides in the flavonols quercetin, isorhamnetin and kaempferol, which have not described previously for this species. The content of total phenolic compounds $\left(8.31 \pm 0.38 \mathrm{mg} \mathrm{g}^{-1}\right.$ of DM) was higher than that of flavonoids $\left(3.08 \pm 0.32 \mathrm{mg} \mathrm{g}^{-1}\right.$ of DM). These results explain the antioxidant activity $\left(\mathrm{IC}_{50}=88.54 \pm 0.15 ; 109.44 \pm 0.48 \mu \mathrm{g}\right.$ $\mathrm{mL}^{-1}$ ) presented by the leaf extracts. The identification of these flavonoids may contribute to the chemotaxonomy of this genus.

Index words: Calia secundiflora, colorin, antioxidant activity, flavonoids,

\section{INTRODUCCIÓN}

El cambio climático ha producido numerosos cambios en la distribución y abundancia de las especies vegetales. Los escenarios futuros predicen el riesgo de extinción de algunas plantas (Thomas et al., 2004). Esto significa una pérdida del potencial que representa el conocimiento de sus metabolitos con propiedades medicinales y de interés agronómico. Por ello existe la necesidad de realizar estudios fitoquímicos y evaluar la actividad biológica de plantas para identificar los principios activos en beneficio del hombre, sobre todo de las especies que no han sido estudiadas. Además, el estudio fitoquímico puede contribuir al conocimiento taxonómico de las especies, como es el caso de Calia secundiflora (Ort.) Yakovlev.

C. secundiflora (Ortega) Yakovlev (Fabaceae) se encuentra distribuida en África, América y Asia (Hatfiled et al., 1977; Shulthes y Hofmann, 1982). En México se localiza en los Estados de Sonora, Chihuahua, Coahuila, Nuevo León, San Luis Potosí, Tamaulipas, Zacatecas, Querétaro e Hidalgo (Rzedowski, 1978; Aguilar y Zolla, 1982), donde 
se le conoce localmente como Patol, Pitol, Coca, Colorín, Chocolón y Frijolillo. Sin embargo, es más conocida como Sophora secundiflora (Ortega) Lag. ex DC. y por sus efectos alucinógenos en rituales de grupos étnicos del suroeste de los Estados Unidos (Murakoshi et al., 1986; Keller, 1975). C. secundiflora se considera una planta tóxica por contener alcaloides del tipo quinolizidínico (Zavala et al., 2006), lo que ha restringido su uso medicinal (Hatfield et al., 1977; Murakoshi et al., 1986).

Los alcaloides identificados en esta especie también se han descrito en unas 65 especies de la familia Leguminosae, en particular en Sophora secundiflora, razón por la cual a C. secundiflora se le clasificó inicialmente como Sophora secundiflora (Omiya et al., 1995). Los análisis filogenéticos de algunos géneros (Styphnobium y Cladrastis), anteriormente representativos del género Sophora, han permitido reclasificarlos mediante sus caracteres fitoquímicos (perfil de flavonoides) y moleculares (secuencia de ADN) (Kite y Pennington, 2003). En contraste, aún existen casos no resueltos, como el de C. secundiflora, debido a que hay incongruencia en la secuencia de ADN (Kite y Pennington, 2003), y porque para su reubicación taxonómica se requieren evidencias fitoquímicas de la presencia de alcaloides, fenólicos y flavonoides (Hatfield et al., 1977; Murakoshi et al., 1986). Se justifica así el seguimiento de la posición de este taxón para contribuir a su relación filogenética, con base en el estudio fitoquímico de otros metabolitos y de la actividad biológica de la especie.

Los estudios publicados sólo se han dirigido hacia la investigación de alcaloides en ambos géneros (Sophora y Calia), pero se desconoce la presencia de flavonoides que pudieran justificar los efectos benéficos que puede aportar la especie. Los compuestos de naturaleza fenólica presentes en hortalizas y frutos contribuyen a dar una fuerte capacidad antioxidante (Prior et al., 1998); por su diversidad y amplia distribución constituyen el grupo más importante de metabolitos con actividad antioxidante natural. Hay evidencias epidemiológicas convincentes que muestran los beneficios de los flavonoides en la salud y de su contribución en retardar algunos procesos degenerativos (Robards et al., 1999). Son compuestos con habilidad para atrapar radicales libres, quelatar metales pesados y modular la actividad de ciertas enzimas, y además se ha observado que los flavonoides poseen propiedades anticancerígenas, cardiotónicas, antitrombóticas, vasculares, que disminuyen el colesterol y contribuyen a la protección del hígado, entre otras (Robards et al., 1999). Con base en lo anterior, el objetivo de esta investigación fue identificar los flavonoides en la hoja de esta especie y evaluar su potencial antioxidante, para contribuir de manera indirecta a una validación de la especie por sus características fitoquímicas dentro del género Calia.

\section{MATERIALES Y MÉTODOS}

\section{Recolecta del material vegetal}

El material vegetal (hojas) se recolectó aleatoriamente de diversos ejemplares adultos en etapa de floración (abril de 2007), de una población ubicada en el transecto El Cardonal-Santuario, Municipio El Cardonal, Estado de Hidalgo, México. Este sitio se ubica en laderas con exposición al SSE y SE con pendiente de $40^{\circ}$, en las coordenadas $20^{\circ} 36^{\prime} \mathrm{LN}$ y $99^{\circ} 07^{\prime} \mathrm{LO}$, a $2130 \mathrm{msnm}$. La muestra (hojas) del Estado de Querétaro también se recolectó aleatoriamente en diversos ejemplares adultos en etapa de floración (mayo 2007), en una población ubicada en el transecto Vizarrón del MonteSan Joaquín, municipio de Cadereyta; este sitio se ubica a $2070 \mathrm{msnm}$, en las coordenadas $20^{\circ} 41^{\prime} \mathrm{LN}$ y $99^{\circ} 49^{\prime} \mathrm{LO}$, en laderas con exposición al SE y pendientes de $45^{\circ}$. Se prepararon dos ejemplares de herbario que fueron identificados por el Dr. Mario Sousa del Instituto de Biología de la Universidad Nacional Autónoma de México, con los números de registro 1155623 y 1155624 . Las hojas de Calia secundiflora se secaron a temperatura ambiente durante tres semanas, y luego en una estufa a $45^{\circ} \mathrm{C}$ por $24 \mathrm{~h}$; después se molieron en un molino Thomas-Wiley® modelo 4 (PA., USA) con un tamiz del número 2.

\section{Preparación del extracto}

De cada muestra de campo se pesó $1 \mathrm{~g}$ de hoja molida y seca, que se disolvió en $25 \mathrm{~mL}$ de etanol a $95 \%$; después de $24 \mathrm{~h}$ se ajustó su volumen a $25 \mathrm{~mL}$ con etanol a $80 \%$, y se centrifugó la mezcla a $2000 \mathrm{rpm}$.

\section{Cuantificación de fenoles totales}

El contenido de fenoles totales se cuantificó con el método propuesto por Waterman y Mole (1994). A $0.5 \mathrm{~mL}$ del extracto etanólico se le adicionó $10 \mathrm{~mL}$ de una solución de $\mathrm{Na}_{2} \mathrm{CO}_{3}$ a $10 \%$ y se incubó a $38^{\circ} \mathrm{C}$ por $15 \mathrm{~min}$. Se tomó 1 $\mathrm{mL}$ de esta mezcla, a la que se le adicionó $3 \mathrm{~mL}$ de agua y $1 \mathrm{~mL}$ del reactivo de Folín-Ciocalteu: agua 1:1 v/v. La mezcla se dejó reposar por $15 \mathrm{~min}$ en oscuridad. Finalmente se tomó lectura de la absorbancia a $660 \mathrm{~nm}$ en un espectrofotómetro Spectronic $20 \circledR$ (Florida, USA). La concentración se calculó a partir de una curva patrón $\left(\mathrm{y}=0.0014 \mathrm{x} ; \mathrm{R}^{2}=\right.$ 0.9979) elaborada con fenol. Cada análisis se efectuó por triplicado. El contenido total de fenólicos en el extracto se expresó en $\mathrm{mg}$ de equivalentes de fenol por $\mathrm{g}$ de materia seca ( $\left.\mathrm{mg} \mathrm{g}^{-1} \mathrm{MS}\right)$.

\section{Cuantificación de flavonoides totales}

A una alícuota de $0.5 \mathrm{~mL}$ del sobrenadante del extracto 
etanólico preparado anteriormente, se le agregó $1.5 \mathrm{~mL}$ de etanol a $95 \%, 0.1 \mathrm{~mL}$ de una solución de $\mathrm{AlCl}_{3}, 0.1 \mathrm{~mL}$ de solución $1 \mathrm{M}$ de acetato de potasio y $2.8 \mathrm{~mL}$ de agua destilada; la mezcla se incubó por $30 \mathrm{~min}$. Su absorbancia se leyó en un espectrofotómetro Spectronic $20 \AA$ a una longitud de onda $415 \mathrm{~nm}$. Para la cuantificación de la concentración se hizo una curva patrón $\left(\mathrm{y}=0.0122 \mathrm{x}-0.0067 ; \mathrm{R}^{2}=0.9653\right)$ preparada con quercetina (Chang et al., 2002). Los resultados fueron expresados en mg equivalentes de quercetina por g de materia seca ( $\left.\mathrm{mg} \mathrm{g}^{-1} \mathrm{MS}\right)$.

\section{Extracción de flavonoides}

El material vegetal $(1 \mathrm{~kg})$ se sometió a un proceso de extracciones exhaustivas $(3 \times 48 \mathrm{~h})$ con metanol $(\mathrm{MeOH})$, seguido de una decantación del disolvente, el cual se concentró a sequedad mediante vacío en un rotaevaporador Bücki R-210® (Slawil, Switzerland) a $45^{\circ} \mathrm{C}$. Se usaron $5 \mathrm{~g}$ del extracto metanólico para hacer su partición con diclorometano: agua $(5 \times 5 \mathrm{~mL})$. La fase acuosa se fraccionó con butanol $(5 \times 5 \mathrm{~mL})$ y la porción butanólica se concentró a sequedad mediante vacío. La detección de flavonoides se efectuó en los extractos metanólico y butanólico por cromatografía en capa fina en gel de sílice 60 F254 (Merck), y como eluyente se utilizó una mezcla de butanol: ácido acético: agua (BAW) en una proporción de 40:10:50 \% (v/v). Las placas cromatográficas se revelaron por aspersión sucesiva de los reactivos NP (ácido 2-amino-etil éster difenilbórico a $1 \%$ en metanol, p/v) y PEG (polietilenglicol 4000 a $5 \%$ en metanol, $\mathrm{p} / \mathrm{v}$ ). Las placas se visualizaron a una longitud de onda de $365 \mathrm{~nm}$. Se observó una fluorescencia de color anaranjado, característica de los flavonoides (Wagner y Bladt, 1996).

\section{Fraccionamiento del extracto butanólico}

Se aplicaron $700 \mathrm{mg}$ del extracto butanólico a una placa preparativa de gel de sílice 60 (Merck) de $2 \mathrm{~mm}$ de grosor; como eluyente se utilizó una mezcla de BAW en la proporción 40:10:50 (\% v/v). Las placas se visualizaron con luz UV a $365 \mathrm{~nm}$ y se revelaron en un extremo con el reactivo NP y PEG a 1 y $5 \%$ en metanol, respectivamente, reactivos específicos para la identificación de flavonoides (Wagner y Bladt, 1996). Posteriormente se rasparon los componentes de la placa y se extrajeron del gel de sílice con metanol. De los componentes se obtuvieron dos fracciones ( 1 y 2 ) que se concentraron a sequedad mediante vacío.

\section{Identificación de flavonoides por CL-EM}

La identificación de flavonoides se efectuó en un cromatógrafo de líquidos Waters ${ }^{\circledR}$ modelo LC600 (Massachusetts, USA) en fase reversa, provisto con un detector de arreglo diodos acoplado a un espectrómetro de masas mo- delo Finnigan ${ }^{\circledR}$ MAT900 XLT (Hemmel Hempstead, U.K). Se utilizó una columna Phenomenex Luna C18 (150 mm x $4.6 \mathrm{~mm}$ de d.i. y tamaño de partícula de $5 \mu \mathrm{m}$ ). Como fase móvil se empleó un gradiente de elución para la mezcla metanol/agua/ACN + $1 \%$ ácido fórmico: 0:90:10 (0'); 40:50:10 (30'); 40:50:10 (40’); 90:0:10 (45’); 90:0:10 (47’); 0:90:10 (55), a una velocidad de flujo de $1 \mathrm{~mL} \mathrm{~min}^{-1} \mathrm{y}$ un volumen de inyección de $20 \mu \mathrm{L}$ del extracto metanólico. Para el detector del espectrómetro de masas se utilizó una trampa de iones cuadrupolo (Finnigan ${ }^{\circledR}$ LCQ, Hemmel Hempstead, U.K) acoplado con una fuente iónica APCI. La temperatura de vaporización fue de $550{ }^{\circ} \mathrm{C}$, y la de calentamiento de la columna capilar de $150^{\circ} \mathrm{C}$. Se utilizó una corriente de nitrógeno y un flujo auxiliar del mismo gas a 80 y 10 unidades, respectivamente. La identificación de los flavonoides en las fracciones estudiadas se llevó a cabo por comparación con muestras auténticas y por cotejo con la biblioteca de espectros del equipo (Cuyckens y Claeys, 2004; Kite et al., 2007).

\section{Evaluación de la actividad antioxidante}

Este análisis se hizo con el método del radical libre DPPH (2,2-difenil-1-picrilhidracilo) descrito por Liu et al. (2009), y solamente en el extracto de hojas proveniente del Estado de Hidalgo, porque las muestras del Estado de Querétaro presentaron una baja concentración de metabolitos en comparación con las de Hidalgo. La capacidad antioxidante de las muestras fue observada a $516 \mathrm{~nm}$ por el cambio de coloración gradual del DPPH (púrpura) a DPPH-reducido (amarillo) (Soler-Rivas et al., 2000; Cotelle et al., 1996).

Se preparó una solución metanólica de DPPH a una concentración $0.1 \mathrm{mM}$. Los extractos $(1 \mathrm{~mL})$ metanólico, butanólico y las fracciones 1 y 2 , se diluyeron con metanol para obtener las concentraciones de 0.2, 0.15, 0.1, $0.05 \mathrm{mg} \mathrm{mL}^{-1}$. A cada concentración de los extractos y de las fracciones se les adicionó $3 \mathrm{~mL}$ de la solución de DPPH $(0.1 \mathrm{mM})$ y se mezclaron separadamente. Las mezclas se dejaron a temperatura ambiente y oscuridad durante 30 min y después se les midió su absorbancia a $516 \mathrm{~nm}$ en un espectrofotómetro Spectronic $20{ }^{\circledR}$. Se determinó el porcentaje de DPPH remanente mediante la fórmula:

$$
\% \mathrm{DPPH}=\left(\mathrm{A}_{\text {blanco }}-\mathrm{A}_{\text {muestra }}\right) \times 100 / \mathrm{A}_{\text {blanco }}
$$

En donde $\mathrm{A}_{\text {blanco }}$ es la absorbancia del testigo (DPPH $0.1 \mathrm{mM}$ ); $\mathrm{A}_{\text {muestra }}$ es la absorbancia obtenida de cada muestra después de $30 \mathrm{~min}$ con DPPH $0.1 \mathrm{mM}$. La actividad antioxidante de las muestras se determinó mediante el cálculo de la concentración inhibitoria media $\left(\mathrm{CI}_{50}\right)$, que es la concentración requerida por la muestra para disminuir la absorbancia del DPPH a $50 \%$. La baja absorbancia de la mezcla de reacción indicó alta actividad antioxidante.

Se construyó una curva estándar de DPPH; para ello 
se pesó $3.93 \mathrm{mg}$ del reactivo y se disolvió en $100 \mathrm{~mL}$ de metanol para obtener una concentración de $0.1 \mathrm{mM}$. De esta solución patrón se prepararon las diferentes concentraciones: $0.01,0.02,0.04,0.06,0.08$ y $0.1 \mathrm{mM}$ de DPPH. La absorbancia se midió a $516 \mathrm{~nm}$ en un espectrofotómetro Spectronic 20®; las lecturas se tomaron por triplicado y como blanco se utilizó metanol.

Para determinar la cinética de reacción de los extractos y de las fracciones 1 y 2 con el DPPH remanente, se siguió el método modificado de Sánchez-Moreno et al. (1999). Se preparó separadamente una solución de $1 \mathrm{mg} \mathrm{mL}^{-1}$ de metanol de cada muestra, de la cual se tomó $0.1 \mathrm{~mL}$ y se le adicionó $3.9 \mathrm{~mL}$ de la solución de DPPH $(5 \mathrm{mg} / 100 \mathrm{~mL}$ de metanol). Posteriormente, se midieron las absorbancias del DPPH con intervalos de $30 \mathrm{~s}$, desde el tiempo cero $\left(\mathrm{DPPH}_{T=0}\right)$ hasta concluir los $30 \mathrm{~min}\left(\mathrm{DPPH}_{\mathrm{rem}}\right)$ en un espectrofotómetro Spectronic $20 \AA$, a una longitud de onda de $515 \mathrm{~nm}$. Los datos obtenidos sirvieron para calcular el DPPH remanente en el medio de reacción. Cada lectura se hizo por triplicado, y como blanco se uso metanol. La concentración de $\mathrm{DPPH}_{\text {rem }}$ se calculó con la ecuación:

$$
\% \mathrm{DPPH}_{\mathrm{rem}}=100 \times \mathrm{DPPH}_{\mathrm{rem}} / \mathrm{DPPH}_{T=0}
$$

\section{Análisis estadístico}

Se efectuó un análisis de varianza y la prueba de comparación de medias (Tukey, 0.05) mediante el programa de cómputo SAS Institute versión 8.0 (SAS Institute, 1999).

\section{RESULTADOS Y DISCUSIÓN}

\section{Cuantificación de fenoles y flavonoides}

De $1 \mathrm{~kg}$ de muestra seca se obtuvo un rendimiento de $10.23 \%$ de extracto metanólico y $1.02 \%$ de extracto butanólico. El fraccionamiento del extracto butanólico por cromatografía en capa fina preparativa permitió obtener 0.8 $\mathrm{mg} \mathrm{g}^{-1}$ y $1.1 \mathrm{mg} \mathrm{g}^{-1}$ de materia seca en las fracciones 1 ( $\mathrm{Rf}$ $=0.26)$ y $2(R f=0.40)$, respectivamente, ya que de acuerdo con el análisis preliminar por cromatografía en capa fina se confirmó cualitativamente la presencia de flavonoides.

El contenido de fenólicos $\left(8.31 \pm 0.38 \mathrm{mg} \mathrm{g}^{-1}\right.$ de MS) fue mayor al de flavonoides (3.08 $\pm 0.32 \mathrm{mg} \mathrm{g}^{-1}$ de MS) en hojas provenientes del Estado de Hidalgo. El contenido total de compuestos fenólicos fue de $5.61 \pm 0.05 \mathrm{mg} \mathrm{g}^{-1}$ de MS y el de flavonoides de $2.76 \pm 0.14 \mathrm{mg} \mathrm{g}^{-1}$ de MS, en hojas de $C$. secundiflora provenientes del Estado de Querétaro.

No se encontraron estudios similares efectuados en especies de este género para poder realizar una comparación. Sin embargo, se infiere que una pequeña proporción del contenido de compuestos fenólicos corresponde a flavonoides, y que el resto podría estar constituido de procianidinas u otros ácidos fenólicos, como sucede en otras especies vegetales según describieron Cui et al. (2006).

Entre regiones no hubo diferencias significativas en el contenido de flavonoides en las muestras de las dos zonas, pero sí la hubo $(\mathrm{P} \leq 0.05)$ en la concentración de fenólicos, aunque el contenido de fenólicos tendió a ser superior en las muestras provenientes de la región más perturbada (Hidalgo). Al respecto, en un estudio comparativo de hábitat de C. secundiflora en los Estados de Hidalgo y Querétaro, García-Mateos et al. (2007) observaron un mayor contenido de alcaloides en las muestras de Hidalgo que en las de Querétaro, lo cual asociaron al grado de perturbación y hábitat de la especie.

El contenido y perfil de metabolitos puede variar con las condiciones climáticas y geográficas. En semillas de soya (Glycine max L.) Caldwell et al. (2005) observaron un incremento en la concentración de isoflavonas al aumentar la temperatura, la concentración de $\mathrm{CO}_{2}$ y el déficit hídrico. Kirakosyan et al. (2004) también encontraron niveles elevados de compuestos fenólicos en hojas de Crataegus laevigata y C. monogyna cuando estuvieron en condiciones de estrés por déficit de agua.

\section{Identificación de flavonoides por CL-EM}

El análisis por cromatografía de líquidos-espectrometría de masas permitió identificar en el extracto metanólico, a siete glicósidos de tres flavonoides: quercetina $(\mathrm{Q})$, kaenferol (K) e isoramnetina (I); a otros compuestos no fue posible identificarlos. En el Cuadro 1 se muestran las características cromatográficas y espectrométricas de los compuestos detectados en el extracto metanólico, y en la Figura 1 se presentan las estructuras de los glicósidos de los flavonoides identificados. Los tiempos de retención y los valores de los iones moleculares de los glicósidos coincidieron con los existentes en la biblioteca de espectros del equipo y con los descritos en la literatura (Kite et al., 2007).

\begin{tabular}{|c|c|c|c|c|c|}
\hline Núm & $t_{\mathrm{R}}(\min )$ & {$\left[\mathrm{M}^{+}\right]$} & Aglicona $^{\dagger}$ & $\mathrm{C}-3^{*}$ & C-7 \\
\hline 1 & 8.53 & 918 & $\mathrm{i}$ & Rha $(1 \rightarrow 2)[$ Rha- $(1 \rightarrow 6)]$-Glc- & Rha- \\
\hline 2 & 12.71 & 901 & $q$ & Rha $(1 \rightarrow 2)[$ Rha- $(1 \rightarrow 6)]$-Glc- & Rha- \\
\hline 3 & 14.75 & 885 & $\mathrm{k}$ & Rha $(1 \rightarrow 2)[$ Rha- $(1 \rightarrow 6)]$-Glc- & Rha- \\
\hline 4 & 17.80 & 755 & $q$ & Rha $(1 \rightarrow 2)[$ Rha- $(1 \rightarrow 6)]$-Glc- & -- \\
\hline 5 & 18.26 & 755 & $\mathrm{q}$ & Rha $(1 \rightarrow 6)-$ Glc- & Rha- \\
\hline 6 & 20.3 & 595 & $\mathrm{k}$ & Rutinósido (Rha $(1 \rightarrow 6)-$ Glc-) & -- \\
\hline 7 & 21.86 & 609 & $\mathrm{q}$ & Rutina & -- \\
\hline
\end{tabular}




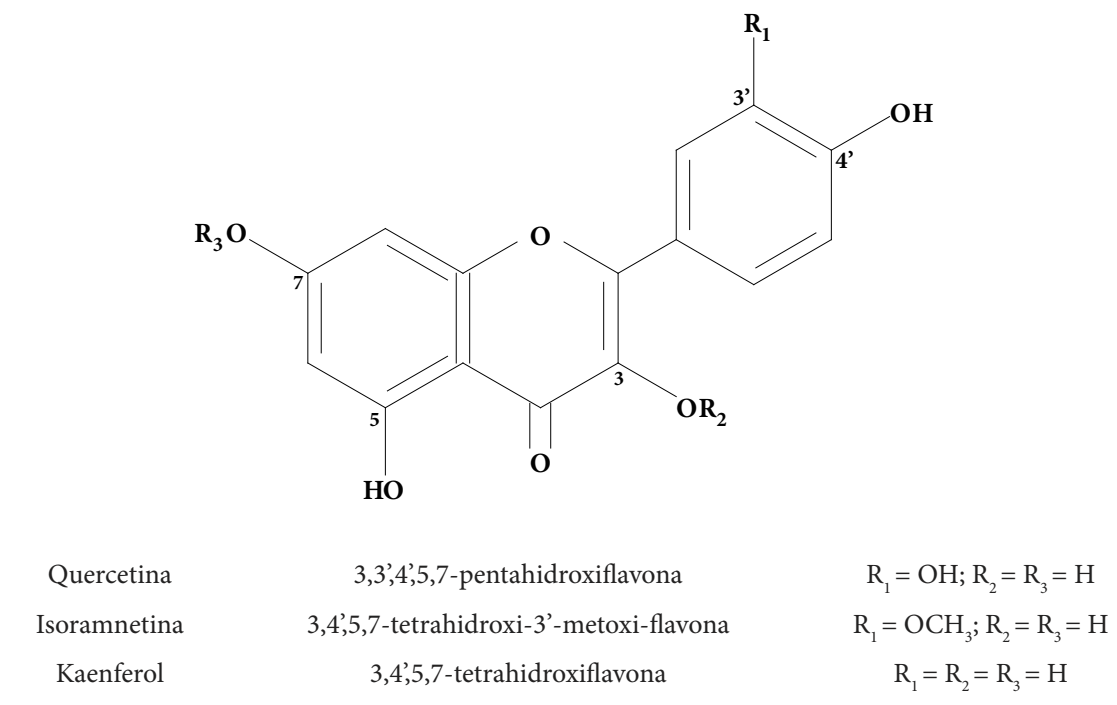

Figura 1. Estructura de las agliconas en los glicósidos identificados en C. secundiflora.

La presencia de di-, tri- y tetraglicósidos de estos flavonoles no se han descrito en la C. secundiflora, especie en la que se ha señalado la presencia de otros flavonoides. Shirataki et al. (1997) describieron tres isoflavonoides aislados de la raíz de C. secundiflora. Por su parte, Tanaka et al. (1998) detectaron ocho nuevos isoflavonoides en la raíz de $S$. secundiflora, S. arizonica y S. gypsophila.

Glicósidos de flavonoides similares a los detectados en este trabajo en C. secundiflora fueron descritos recientemente en otras leguminosas y han servido como marcadores. Kite et al. $(2007,2009)$ describieron la presencia de glicósidos de flavonoides en especies del género Styphnolobium y en la especie Cladrastis kentukea. Kite et al. (2007) identificaron a los mismos glicósidos en la especie Styphnolobium japonicum, que en el presente estudio se muestran en el Cuadro 1 como estructuras 3, 4, 6 y 7.

\section{Actividad antioxidante}

Se encontraron diferencias significativas $(\mathrm{P} \leq 0.05)$ en la concentración inhibitoria media $\left(\mathrm{CI}_{50}\right)$ entre los extractos metanólico, butanólico y las fracciones 1 y 2 para reducir a $50 \%$ el DPPH (Cuadro 2). Los extractos butanólico y metanólico presentaron mayor actividad antioxidante que las fracciones aquí detectadas.

La cinética de reacción mostró que la cantidad de DPPH remanente disminuyó por la actividad antirradical (actividad antioxidante) de los extractos y de las fracciones evaluadas (Figura 2). Sánchez-Moreno (2002) clasificó el desempeño antioxidante de varias sustancias de acuerdo con la cinética de la reacción, como: $<5 \mathrm{~min}$, rápida; entre 5 y 30 min, intermedia; y $>30$ min como lenta. Para este caso,
Cuadro 2. Concentración inhibitoria media $\left(\mathrm{CI}_{50}\right)$ de $\mathrm{DPPH}$ (2,2-difenil-1-picrilhidracilo) con los extractos y fracciones de Calia secundiflora.

\begin{tabular}{cc}
\hline Extracto & $\begin{array}{c}\mathrm{CI}_{50} \\
\left(\mu \mathrm{gL}^{-1}\right)\end{array}$ \\
\hline Extracto metanólico & $109.443 \pm 0.482 \mathrm{c}$ \\
Extracto butanólico & $88.543 \pm 0.151 \mathrm{~d}$ \\
Fracción 1 & $568.630 \pm 1.152 \mathrm{a}$ \\
Fracción 2 & $168.903 \pm 1.277 \mathrm{~b}$ \\
\hline $\mathrm{CI}_{50}$ fue determinada como la concentración requerida de las \\
muestra para inhibir en 50 \% al DPPH en 30 min. Datos con la \\
misma letra son estadísticamente iguales (Tukey, 0.05). Después \\
de cada valor se presentan el error estándar $(\mathrm{n}=3)$.
\end{tabular}

los extractos metanólico y butanólico tuvieron una cinética más rápida que las muestras restantes, y también dieron una menor concentración de DPPH remanente a los $30 \mathrm{~min}$, en congruencia con los resultados del Cuadro 2; estos dos extractos fueron los de mayor actividad antioxidante debido al efecto sinérgico de los glicósidos en la mezcla.

La actividad antioxidante de los extractos metanólico y butanólico de C. secundiflora se debe, en parte, a la presencia de los siete glicósidos en quercetina, de isoramnetina y de kaenferol. Se ha documentado ampliamente que la presencia de estos metabolitos se asocia con la actividad antioxidante de una amplia variedad de frutos y otras especies (Chang et al., 2002; Harborne y Williams, 2000; Robards et al., 1999). La actividad antioxidante de flavonoles y flavonas se asocia con sus características estructurales. Las estructuras que poseen grupos hidroxilos en las posiciones $3^{\prime}$ y 4 ' del anillo B y OH en C-3 e insaturación del anillo C, permiten estructuras mesoméricas estables con capacidad eficiente de captura de radicales libres, requisito para una máxima capacidad antioxidante (Robards et al., 1999; Cotelle et al., 1996; Bors et al., 1990). En este caso, la quercetina cumple en mayor medida con estas características estructurales, 


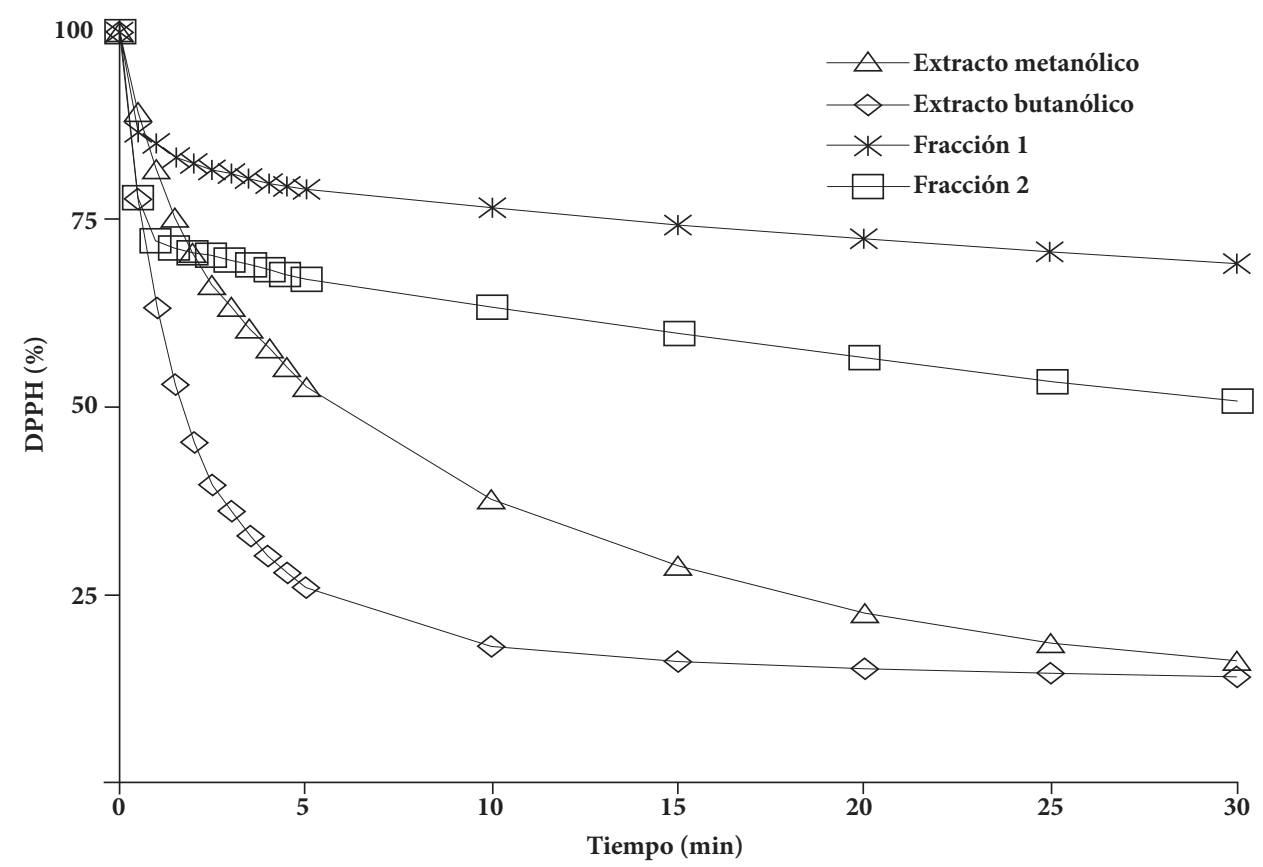

Figura 2. Actividad antioxidante de los extractos y fracciones de $C$. secundiflora en relación con la variación de la concentración de DPPH (2,2-difenil-1-picrilhidracilo) remanente en función del tiempo.

aunque se ha descrito que algunos de sus derivados glicosídicos presentan menor actividad antioxidante que la aglicona por la unión del azúcar al OH del C-3 (Robards et al., 1999). La actividad antioxidante aquí encontrada se explica por la estructura de los glicósidos detectados en los extractos y en las fracciones.

\section{CONCLUSIONES}

En las dos Estados muestreados, Hidalgo y Querétaro, el contenido total de compuestos fenólicos en hojas de Calia secundiflora fue mayor que el de flavonoides, aunque en las muestras de Hidalgo tendieron a ser mayores, posiblemente por ser una zona perturbada. Mediante el análisis de CLEM se identificaron siete di-, tri- y tetra glicósidos descritos por primera vez en esta especie. La presencia de glicósidos en los flavonoles quercetina, isoramnetina y kaenferol, explican la actividad antioxidante encontrada en los extractos $\mathrm{y}$ en las fracciones de flavonoides de hojas provenientes del Estado de Hidalgo. Estos resultados pueden contribuir a la quimiotaxonomía de C. secundiflora y al conocimiento fitoquímico de las especies mexicanas, y en la distribución de nuevos principios activos.

\section{BIBLIOGRAFÍA}

Aguilar C A, C Zolla (1982) Plantas Tóxicas de México. Instituto Mexicano del Seguro Social. México, D. F. 271 p.

Bors W, W Heller, C Michel, S Manfred (1990) Flavonoids as antioxidants: determination of radical-scavenging efficiencies. Meth. Enzymol.
186:343-355.

Caldwell C R, S Britz, R Mirecki (2005) Effect of temperature, elevated carbon dioxide, and drought during seed development on the isoflavone content of dwarf soybean [Glycine max (L.) Merrill] grown in controlled environments. J. Agric. Food Chem. 53:11251129.

Chang C, M Yang, H Wen, J CHern (2002) Estimation of total flavonoids content in propolis by two complementary colorimetric methods. J. Food Drug Anal. 10:176-182.

Cotelle N, J Jery, J Wallet, E M Gaydou (1996) Antioxidant properties of hidroxy-flavones. Free Radical Biol. Med. 20:35-43.

Cui N, K Nakamura, S Tian, H Kayahara, Y Tian (2006) Polyphenolic content and physiological activities of Chinese hawthorn extracts. Biosci. Biotechnol. Biochem. 70:2948-2956.

Cuyckens F, M Claeys (2004) Mass spectrometry in the structural analysis of flavonoids. J. Mass Spectrom. 39:1-15.

García-Mateos R, M Soto-Hernández, F Zavala-Chávez, G Kite (2007) Quinolizidine alkaloids in Calia secundiflora (Leguminosae). Agrociencia 41:161-167.

Harborne J B, C Williams (2000) Review advances in flavonoid research since 1992. Phytochemistry 55:481-504.

Hatfield G M, L J Valdes, W Keller, W Merril, V Jones (1977) An investigation of Sophora secundiflora seeds (Mescalbeans). Lloydia 40:374-383.

Keller W J (1975) Alkaloids from Sophora secundiflora. Phytochemistry 14:2305-2306.

Kirakosyan A, P Kaufman, S Warber, S Zick, K Aaronson, S Bolling, S Ch Chang (2004) Applied environmental stresses to enhance the levels of polyphenolics in leaves of hawthorn plants. Physiol. Plant.121:182-186.

Kite G C, Ch Stoneham, N Veitch (2007) Flavonol tetraglycosides and other constituents from leaves of Styphnolobium japonicum (Leguminosae) and related taxa. Phytochemistry 68:1407-1416.

Kite G, N C Veitch, M E Boalch, G P Lewis, C J Leon, M S J Simmonds (2009) Flavonol tetraglycosides from fruits of Styphnolobium japonicum (Leguminosae) and the authentication of fructus Sophorae and flos Sophorae. Phytochemistry 70:785-794. 
Kite G, R Pennington (2003) Quinolizidine alkaloid status of Styphnolobium and Cladrastis (Leguminosae). Biochem. Syst. Ecol. 31:1409-1416.

Liu L, Y Laura, T Liang, X Ye Hong, X Zeng (2009) Determination of polyphenolic content and antioxidant of kudingcha made from Ilex kudingcha C. J. Tseng. Food Chem. 112:35-41.

Murakoshi I, H Kubo, M Ikram, M Israr, N Shafi, S Ohmiya, H Otomasu (1986) (+)-11-oxocytisine, a lupin alkaloid from leaves of Sophora secundiflora. Phytochemistry 25:2000-2002.

Prior R L, G Cao, A Martin, E Sofic, J Mcewen, C O’brien, N Lischner, M Ehlenfeldt, W Kalt, G Krewer, C Mainland (1998) Antioxidant capacity as influenced by total phenolic and anthocyanin content, maturity, and variety of Vaccinium species. J. Agric. Food Chem. 46:2686-2693.

Robards K, D Prenzler, G Tucker, P Swatsitang, W Glover (1999) Phenolic compounds and their role in oxidative processes in fruits. Food Chem. 66:401-436.

Rzedowski J (1978) Vegetación de México. Ed. Limusa. México, D. F. 432 p.

Sánchez-Moreno C (2002) Methods used to evaluate the free radical scavenging activity in foods and biological systems. Food Sci. Technol. Internt. 8:121-137.

Sánchez-Moreno C, J A Larrauri, F Saura-Calixto (1999) Free radical scavenging capacity and inhibition of lipid oxidation of wines, grape juices and related polyphenolic constituents. Food Res. Internt. 32:407-412.
Shirataki Y, Y Sanae, Y Sugita, I Yokoe, M Komatsu, M Ohyama, T Tanaka, M Iinuma (1997) Isoflavanones in roots of Sophora secundiflora. Phytochemistry 44:715- 718 .

Shulthes R E, A Hofmann (1982) Plantas de los Dioses: Origen y Uso de los Alucinógenos. México. Fondo de Cultura Económica. $192 \mathrm{p}$.

Soler-Rivas C, J C Espín, H J Wichers (2000) An easy and fast test to compare total free radical scavenger capacity of foodstuffs. Phytochem. Anal. 11:330-338.

Tanaka T, M Ohyama, M Iinuma, Y Shirataki, M Komatsu, Ch L Burandt (1998) Isoflavonoids from Sophora secundiflora, S. Arizonica and S. Gypsophila. Phytochemistry 48:1187 1193.

Thomas C D, A Cameron, R E Green, M Bakkenes, L J Beaumont, Y C Collingham, B F N Erasmus, M Ferreira de Siqueira, A Grainger, L Hannah, L Hughes, B Huntley, A S Van Jaarsveld, G F Midgley, L M Miles, M A Ortega-Huerta, A T Peterson, O L Phillips, S E Williams (2004) Extinction risk from climate change. Nature 427:145-148.

Wagner H, S Bladt (1996) Plant Drug Analysis. A Thin Layer Chromatography Atlas. Springer Verlag, Berlin. 384 p.

Waterman P G, S Mole (1994) Analysis of Phenolic Plant Metabolites. Methods in Ecology. Blackwell Scientific Publications. Oxford, UK. 238 p.

Zavala C F, R García M, M Soto H, G Kite (2006) Phytochemical differences between Calia secundiflora (Ort.) growing at two sities in Mexico. Z. Naturforstch. 61:155-159. 